\title{
Design and optimization of knee varus vibration orthopedic rehabilitation correction mechanism control system
}

\author{
BaiYan $^{1, a}$,Zheng Wengang ${ }^{2, \mathrm{~b}}$, Zhou Jingbo ${ }^{3, c}$ \\ ${ }^{1}$ BeiHua University Engineering Training Center \\ ${ }^{2}$ Beihua University Mechanical Engineering College \\ ${ }^{3}$ Funing Qinhuangdao district power supply company \\ abyw1689@sohu.com, b745575912@qq.com
}

Keywords: Rehabilitation system; correction; single chip microcomputer

\begin{abstract}
This paper presents a new design concept for the single treatment of X/O type lower limb rehabilitation instrument, the new design concept, modeling and simulation using SOLIDWORKS, the force analysis using ADAMS, control the use of single-chip microcomputer, the real realization of a set of rehabilitation, massage and promoting blood circulation in one of the functions of the machine, and has a very broad market application prospect.
\end{abstract}

\section{Introduction}

At present, the domestic and international treatment of the knee inside and outside of the device is less, and the function of the accuracy is poor.Along with the progress of the society and the development of economy, the development of orthopedic rehabilitation equipment is essential,at the same time, because of the improvement of people's living standards and the rapid development of physical exercise and other projects,people pay more attention to exercise and recreation,but excessive exercise, the body may appear deformation,in addition, due to the long-term bad posture or incorrect force habits caused by the imbalance of muscle mechanics,long duration of muscular imbalance can lead to the occurrence of a shift in the joints, but also prone to the formation of the knee [1],so it is very important to develop the instrument for the rehabilitation of the instrument.

\section{The hardware structure design of $\mathrm{X} / \mathrm{O}$ type leg rehabilitation instrument}

Internal and external knee turned orthopedic rehabilitation bed for vibration of the mechanical structure is mainly composed of five parts -- part of the stretch, stretching, bed, vibration and correction.

\section{A. Composition of Correctional Institution}

The mechanical components of the correction unit are mainly composed of an overload protection device, a shaft, a correction contact, a supporting plate, a sliding block and a linear guide,Under the action of the clamping force, the knee joint of the knee joint in the longitudinal tension, so that the gap between the knee joints, long-term role in promoting the patient's ligaments on both sides of the force gradually restore balance, so as to reduce or even eliminate the gap between patients with knee joint.

\section{B. Functional requirements of corrective mechanism}

(1) the correction unit is carried out for the three time.

a.The default value is $\mathrm{F} 1=5 \mathrm{~N}$, the duration is 10 minutes, the reset of the corrective contact is second times of the clamping;

b.The pressure value of $\mathrm{F} 2=10 \mathrm{~N}$, the duration of 10 minutes, the reset of the corrective contact, 
third times of clamping;

c.The pressure value of the pressure value of $F 3=15 \mathrm{~N}$, the duration of 10 minutes, the reset of the corrective contact, the operation of the vibration unit.

(2) traction unit

The traction unit can moderate the leg in the leg, in order to coordinate with the correction mechanism.

(3) the vibration relieves muscle pressure

\section{Control system design}

In order to adapt to the actual needs of the rehabilitation medicine and medical auxiliary apparatus, the control system must meet the following technical requirements:

(1) the maximum correction range: moderate $18^{\circ} \sim 27^{\circ}$;

(2) orthopedic force: 7 17N (maximum correction force);

(3) traction: $70 \mathrm{~N}$ (maximum traction);

(4) vibration frequency: $20 \sim 40 \mathrm{~Hz}$;

(5) electric push rod: rated voltage $24 \mathrm{~V}$, stroke $100 \mathrm{~mm}$, speed $1 \mathrm{~mm} / \mathrm{s}$, load $200 \mathrm{~N}$; rated voltage $24 \mathrm{~V}$, stroke $200 \mathrm{~mm}$, speed $1 \mathrm{~mm} / \mathrm{s}$, load $200 \mathrm{~N}$.

\section{A.Signal conditioning unit}

Signal conditioning, including the pressure signal conditioning and tension signal conditioning, etc., The circuit is based on the instrument amplifier circuit, and the BAV99M transistor is used to design an ideal signal conditioning circuit.BVA99 is a switching diode,by the deadline or change by the deadline for conducting the time required shorter than the general diode,BAV99M transistor made of this kind of transistor tube is mainly used in electronic equipment of switch circuit, detection circuit, high-frequency and pulse rectifier circuit and automatic control circuit, in IC network supermarket and more detailed pictures of products, size, package, PDF and other data. In the control system, the pressure signal conditioning circuit and the tension signal conditioning circuit design scheme, the signal conditioning circuit is shown in figure 1.

Because TLC2274 is the 4 independent operational amplifier integrated on the same chip,the instrument amplifier which is composed of TLC2274 has a unified power supply, which is beneficial to the power supply noise and improve the performance of the circuit.In addition, the use of TLC2274 to constitute the instrumentation amplifier to the PCB layout, wiring, and also save the circuit board space.
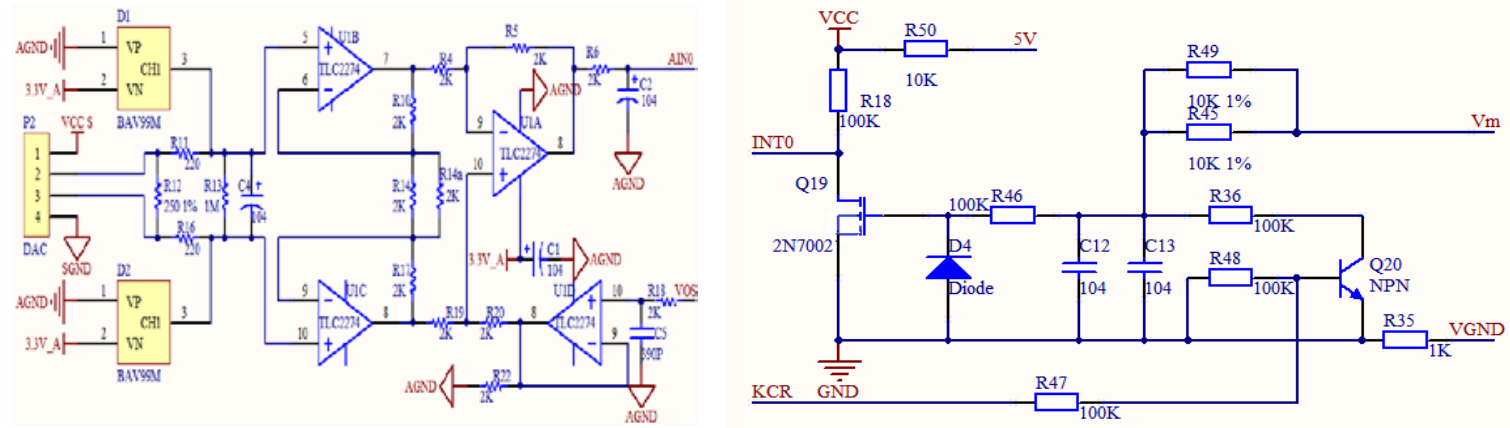

Fig.1. Signal conditioning circuit

Fig.2. Vibration frequency detection circuit 


\section{B. Vibration frequency detection unit}

The relative pressure and tension unit of the vibration element is relatively low, which generally does not cause the harm to the patients,but the frequency is too high, it may cause the patient to feel uncomfortable,designed to be the frequency of numerical visualization, easy to understand the most suitable for their own vibration frequency, so that the next use can be directly adjusted to the last frequency [2].The system uses the following frequency detection circuit, as shown in Figure 2.

\section{Pressure sensor unit}

The control system needs to detect the pressure value of the patient's legs in real time in the process of treatment,reflect the system to control the calibration and display to control platform to help patients and medical staff,after evaluation, the measured value in the $0 \sim 50 \mathrm{~N}$ can be, according to the sensor analysis of the market, can be used in South Korea Shin CBFS-5kg Bong pressure (weighing) sensor.

According to the application requirements of the sensor, the power distribution system based on $78 \mathrm{M} 12$ is designed.78M12 is the three terminal regulator IC integrated circuit, which is only three pin output, respectively, the input, the ground and the output end. Output parameters are $12 \mathrm{~V}$, 500mA.Power distribution circuit of the system is shown in Figure 3.

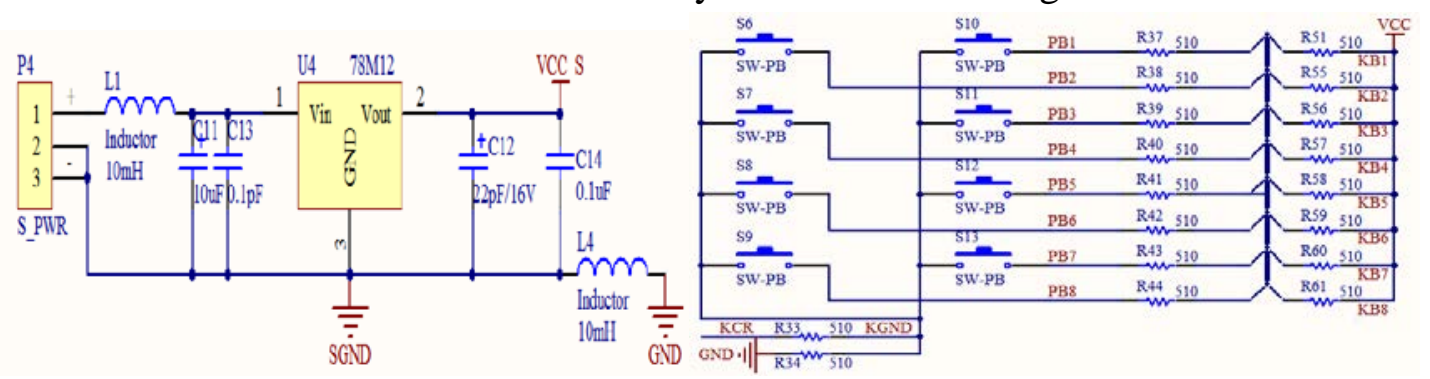

Fig.3. Sensor distribution circuit Fig.4. Regulating keyboard circuit

\section{Software system design}

Because of the different needs of patients to treatment,the control system needs to be controlled by the patient's willingness to reset the correction parameters, so that the treatment effect is more satisfied.In manual mode, the patient can carry out the control of the point and the long-term control. In a certain range, the magnitude of the correction force and traction force, the action time, the vibration frequency and the vibration time of the unit [3].

A.parameter setting section

Parameter setting section of the keyboard circuit as shown in figure 4.

The S7 and S8, S11 and S12, S9 and S13 were used to set the correction force, traction force and vibration frequency parameter values, as well as the preset working time. S6 and S10 as the auxiliary keys, can be short press, long press and long press, used to expand the key functions, to achieve the rectification work, traction work, vibration control and work mode set.

B.Digital tube display circuit and key detection

Digital tube display circuit and key detection circuit shown in figure 5. 


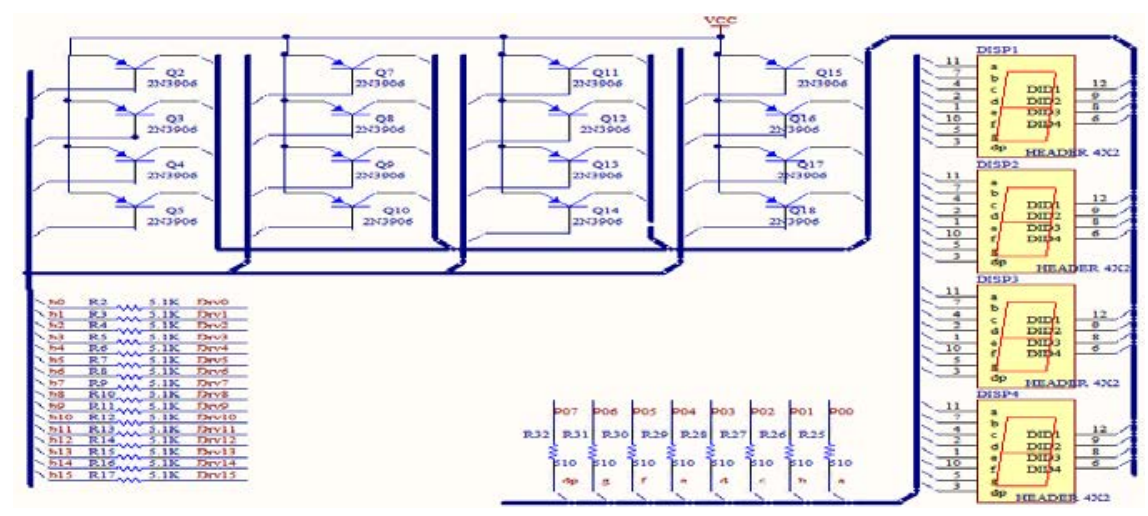

Fig.5. Digital tube display circuit and key detection circuit

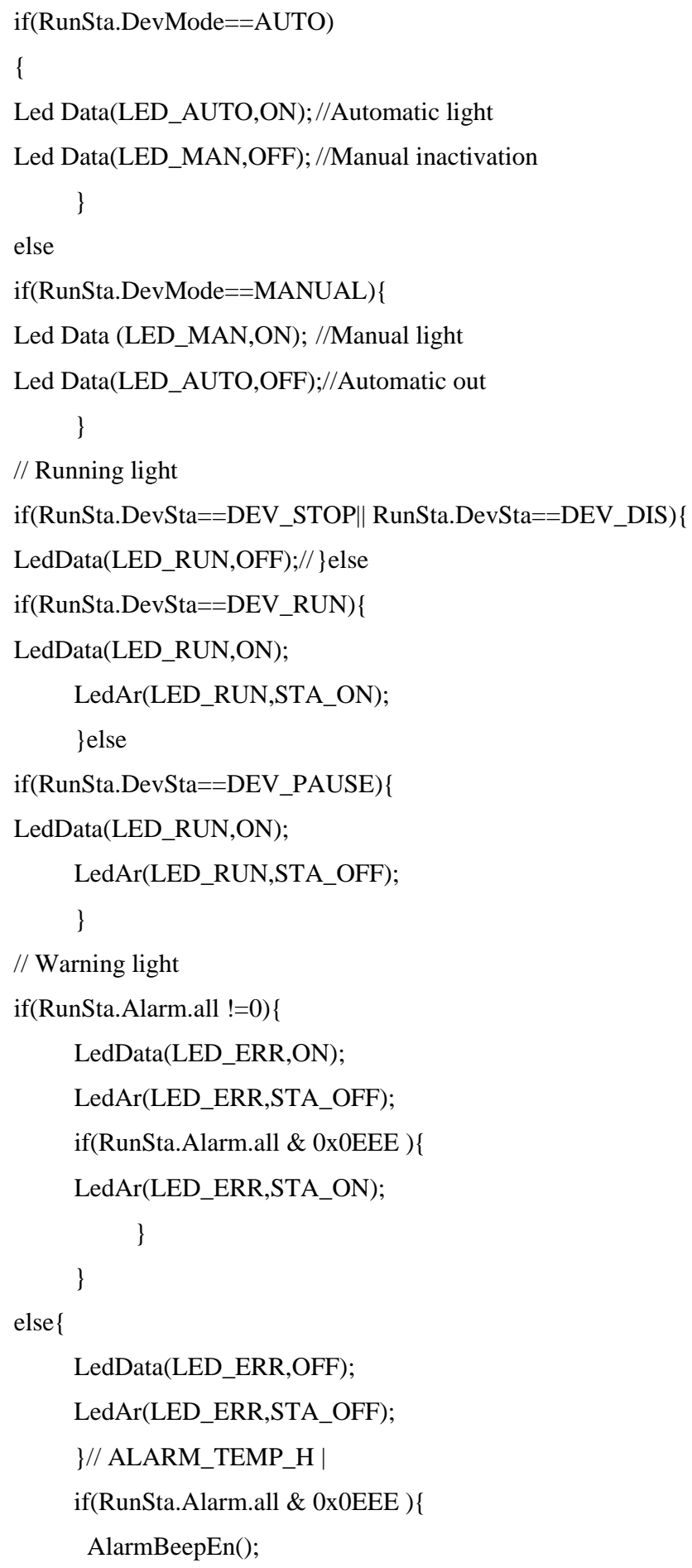


\}else \{

AlarmBeepDis();

\}

\section{Conclusion}

In order to better adapt to the needs of different people, we use semi automatic control,can rely on their own conditions for manual control switch, the control system to adjust the vibration frequency and amplitude,as well as the tension and correction of the patients in the allowable range,the sensor monitoring. The instrument has a certain degree of flexibility and adaptability, the main force according to different patients to set parameters, turn the orthopedic rehabilitation settings in order to complete the knee and, finally reach the purpose of treatment.

\section{References}

[1] Zhang Tie, Xie Cunxi, Zhou Huiqiang, et al. A robotic multifunctional nursing bed and its control system [J]. Journal of South China University of Technology (NATURAL SCIENCE EDITION), 2006.34 (2): 47-51.

[2] Wang Jinhai, Yao Yinhua, Zheng Yu et al. Design of medical care bed system based on SOC microcontroller [J] Journal of Tianjin University of Technology, 2006.25 (4): 44-47

[3] Xie Cunbo, Zhang Dong, Wu Jian. Research and development of robot technology and application, 2003, (6): 21-25

[4] Jiang Shengyuan, Hu Yanjuan, Wang Yao, Li Jianyong based on MCS - 51 single chip microcomputer intelligent multi-function electric rehabilitation bed control system design [J]. mechatronics, 2008.[6]: 42-45 